\title{
Grain Emergency Vehicle Scheduling Problem with Time and Demand Uncertainty
}

\author{
Jiang DongQing' and Zhu QunXiong ${ }^{2}$ \\ ${ }^{1}$ School of Science, Beijing University of Chemical Technology, Beijing 100029, China \\ ${ }^{2}$ College of Information Science \& Technology, Beijing University of Chemical Technology, Beijing 100029, China \\ Correspondence should be addressed to Jiang DongQing; jiangdq@mail.buct.edu.cn
}

Received 8 February 2014; Revised 12 July 2014; Accepted 15 July 2014; Published 20 August 2014

Academic Editor: X. Zhang

Copyright (c) 2014 J. DongQing and Z. QunXiong. This is an open access article distributed under the Creative Commons Attribution License, which permits unrestricted use, distribution, and reproduction in any medium, provided the original work is properly cited.

\begin{abstract}
Grain transportation plays an important role in many relief and emergency supply chains. In this paper, we take the grain emergency vehicle scheduling model between multiwarehouses as the research object. Under the emergency environment, the aim of the problem is to maximize the utilization of vehicles and minimize the delay time. The randomness of the key parameters in grain emergency vehicle scheduling, such as time and demand, is determined through statistical analysis and the model is solved through robust optimization method. Besides, we apply the numerical examples for experimental analysis. We compare the robust optimization model with classic model to illustrate the differences and similarities between them. The results show that the uncertainty of both time and demand has great influence on the efficiency of grain emergency vehicle scheduling problem.
\end{abstract}

\section{Introduction}

In recent years, a lot of large-scale public emergencies occurred frequently, such as the outbreak of SARS in 2003, the Wenchuan earthquake in China in 2008, and the Fukushima nuclear explosion in Japan in 2011, which not only resulted in huge losses, but also left a grieving memory in people's mind. With the rapid development of modern society, social problems of the population, resources, and environment become increasingly acute, leading kinds of public emergencies to occur more frequently. What is worse, the affected scope becomes much wider. Therefore, it is very significant to carry out the grain emergency scheduling effectively, which can protect the safety of people's life in emergencies. How to improve the capability of grain emergency scheduling for the government and enterprises has also become an important topic.

Vehicle scheduling problem is a kind of combinatorial optimization problem widely used in the fields of transportation, logistics, distribution, and so forth. In the process of vehicle service, we may encounter a variety of unexpected events, which will lead to the uncertainty of information, that is, the emergency vehicle scheduling problem, while, in this paper, we will mainly research the grain emergency vehicle scheduling problem. It requires us to adjust the determined parameters according to certain rules, with the uncertainties fully considered. In this case, the deterministic theory and method can no longer deal with these problems. We need to study a new set of theories and methods of vehicle scheduling problem with uncertain information.

At present, the research on emergency logistics is relatively small, while most scholars study the qualitative problem, such as emergency mechanism, the support of emergency materials, and the enactment of emergency laws and regulations. Studies on grain emergency vehicle scheduling are even less. The distribution and transportation of emergency materials especially grain is an important part of the research of emergency logistics, so is the implementation phase of the emergency logistics. Ordinary grain vehicle scheduling problem mainly considers the cost savings. However, the grain emergency vehicle scheduling pays more attention to improving the utilization rate of vehicle efficiency and reducing the delay time with all the constraints met.

In contrast, in this paper we will establish a grain emergency vehicle scheduling model, in which time and demand 
are uncertain. Under the emergency environment, the aim of the problem is to maximize the utilization of vehicles and minimize the delay time. We assume that both the time and demand uncertainty belongs to a box uncertainty. After determining the uncertainty of time and demand, we establish the vehicle scheduling model with parameters determined and undetermined. Then we use the branch and bound algorithm and the robust optimization method to solve the problems. Finally, we list the numerical examples for experimental analysis. The results show that the uncertainty of both time and demand has great influence on the efficiency of grain emergency vehicle scheduling problem. Through the analysis of the data, we will provide the theoretical basis for the practical decision.

The rest of this paper is organized as follows. Section 2 is a brief review of the relevant literature on grain emergency vehicle scheduling. In Section 3, we present a model for grain emergency vehicle scheduling problem. We establish the vehicle scheduling model with parameters determined and undetermined. Finally, we assume that both the time and demand uncertainty belongs to a box uncertainty. We describe a computational study in Section 4. We compare the robust solution against the deterministic solution and then illustrate the effect of various system parameters on the objective function, such as time and demand. We conclude the paper with Section 5 and point out the direction for further research.

\section{Literature Review}

Because of the existence of uncertain factors and multiple objectives, such as the number of vehicles, the latest arrival time and other constraints, the modeling and solving of the grain emergency vehicle scheduling problem is very complicated. At present, there are few studies on this problem, which cannot compare with its importance and current application requirements. Besides, most literature studies the framework of commercial vehicle scheduling problem, without considering some special needs generated by the specific scene of the emergency. For example, the objective function of most literature is to minimize the total cost, while the grain emergency vehicle scheduling problem is to satisfy the needs and reduce the delay time.

Problems where a set of vehicles with finite capacity have to be scheduled at minimum cost are known as the vehicle scheduling problem. This class of problem was introduced by Dantzig and Ramser [1]. Rathi et al. [2] adopt the traditional optimization algorithm for the assignment problem with LP model, but the result is easy to fall into locally optimal solution. This method has strong limitations for the emergency material distribution problem. F. Fiedrich et al. [3] study the problem with time, the quantity of relief goods, and other resources being limited. In their studies, they improve the quality of emergency relief through the effective use of resource, with the minimum of deaths as the objective function. Finally, they put forward the optimal planning model of a plurality of disaster area distribution and emergency relief materials transportation after the earthquake.
Recently, Morales [4] uses robust optimization for the vehicle routing problem with stochastic demands (VRPSD). It is assumed that vehicles replenish at the depot, the worstcase value for the recourse action is computed by finding the longest path on an augmented network, and the problem is solved using a tabu search heuristic.

Using a robust optimization idea to solve uncertain linear optimization problem was first proposed by Soyster [5]. Although its method is too conservative, it is a kind of new train for the study of uncertainty optimization problem, and it is an optimizing condition based on the so-called worst-case, which has caused many scholars' attention and has established foundation for the rapid development of the robust optimization theory. J. M. Mulvey et al. [6] for the first time put forward the concept of robust optimization and applied it to repast problem, power capacity expansion, matrix balance, image reconstruction, and so on.

The robust optimization assumes that uncertain parameters belong to a given bounded uncertainty set. Ben-Tal and Nemirovski [7] propose that a LP with uncertain parameters belonging to a polyhedral uncertainty set has a robust counterpart problem. It is a LP whose size is polynomial in the size of the original problem, and the uncertainty can result in NP-hard problems. With the robust optimization ideas spreading unceasingly, the foundation work is considered by Ben-Tal and Nemirovski $[8,9]$, L. EI Ghaoui et al. [10, 11], and Bertsimas et al. [12-14].

Most research has no detailed introduction on scheduling model and scheduling route planning. At the same time, because of information under uncertainty, the demand of emergency supplies and transportation time cannot be accurately predicted. Hence scheduling model needs to be further researched and developed under uncertainty.

In contrast, in this paper we mainly use robust optimization for the grain emergency vehicle scheduling problem. Our paper differs from most of the existing literature in the following ways: (1) the objective function is to maximize the utilization of vehicles and minimize the delay time, while most of the literature aims to minimize the transportation cost or unmet demand; (2) we assume that both time and demand uncertainty belongs to a box uncertainty, in contrast with most of the stochastic models.

\section{Grain Emergency Vehicle Scheduling Model}

In this section, we first identify the grain emergency vehicle scheduling model. The objective function is to maximize the utilization of vehicles and minimize the delay time. Then we present the uncertainty set and the robust counterpart model. Finally, we propose the time and demand uncertainty belonging to a box uncertainty set.

\subsection{Identifying the Grain Emergency Vehicle Scheduling Model.} Now, consider an emergency vehicle routing problem among several grain warehouses. Problem can be described as follows: there is a batch of relief grain from different warehouses for different demand nodes. Each warehouse has a number of the same type of cars, which start from the warehouse issued, 
leave immediately after arriving at the demand nodes, and eventually return to the warehouse. Besides, each car can only go to a demand node up to one time. If the time of arrival in the demand node is greater than the latest served time, this will cause delay in time. The objective function is to maximize the utilization of vehicles and minimize the delay time.

Parameters. The parameters are as follows:

$m$ : the total number of warehouses,

$n$ : the total number of demand nodes,

$q$ : the total number of vehicles,

$A$ : the set of $m$ grain warehouses and n demand nodes,

$C$ : the set of $m$ grain warehouses,

$D$ : the set of $n$ demand nodes,

$K$ : the set of $q$ vehicles,

$k_{i}$ : the number of vehicles available in warehouse $i$,

$s_{i}$ : the amount of relief supplies provided by warehouse $i$,

$c_{k}$ : the capacity of vehicles $k(k=1, \ldots, q)$,

$r_{k}$ : utilization rate of vehicle $k(k=1, \ldots, q)$,

$d l_{i}$ : the latest time relief grain arrived at demand node $i$, after which more wounded may appear $(i=$ $1, \ldots, n)$,

$t_{i j k}$ : the travel time between node $i$ and node $j$ for vehicle $k$,

$d_{i}$ : the demand at each node $i(i=1, \ldots n)$,

$\gamma$ : the weight of the utilization of vehicles.

Decision Variables. The decision variables are as follows:

$$
x_{i j k}= \begin{cases}1, & \text { if vehicle } k \text { goes from node } i \text { to node } j \\ 0, & \text { otherwise }\end{cases}
$$

$y_{i k}$ : the amount of relief supplies transported to node $i$ by vehicle $k$,

$T_{i k}$ : the time vehicle $k$ arrives at node $i$. We ignore the waiting and loading time after the vehicles arrive at demand nodes, regarding them as 0 or a very small constant,

$\delta_{i k}$ : the delay time of vehicle $k$ for delivering goods at node $i$. If vehicle $k$ arrives at node $I$ before $d l_{i}$, it is equal to 0 . Otherwise, it is more than 0 .
Objective Function. Consider the following:

$$
\begin{aligned}
& \max z=\gamma * \sum_{k \in K} \sum_{i \in D} \frac{y_{i k}}{c_{k}}-\sum_{k \in K} \sum_{i \in D} \delta_{i k}, \\
& \sum_{k \in K} \sum_{j \in D} x_{i j k}<k_{i} \quad \forall i \in C, \\
& \sum_{k \in K} \sum_{j \in A} x_{i j k} \geq 1 \quad \forall i \in D, \\
& \sum_{i \in C} \sum_{j \in D} \sum_{k \in K} x_{i j k}=\sum_{j \in D} \sum_{i \in C} \sum_{k \in K} x_{j i k} \\
& \sum_{i \in A} x_{i j k}=\sum_{i \in A} x_{j i k} \leq 1 \quad \forall j \in D, k \in K, \\
& x_{i j k}+x_{j i k} \leq 1 \quad \forall i \in D, j \in D, k \in K \text {, } \\
& T_{i k}=\delta_{i k}=0 \quad \forall i \in C, k \in K, \\
& M *\left(x_{i j k}-1\right)<T_{i k}+t_{i j k}-T_{j k}<M *\left(1-x_{i j k}\right) \\
& \forall i \in C, \quad j \in D, \quad k \in K, \\
& M *\left(x_{i j k}-1\right)<T_{i k}+t_{i j k}-T_{j k}<M *\left(1-x_{i j k}\right) \\
& \forall i \in D, \quad j \in D, \quad k \in K \\
& 0<T_{i k}-\delta_{i k}<d l_{i} * \sum_{j \in A} x_{i j k} \quad \forall i \in D, k \in K, \\
& \delta_{i k}<\sum_{j \in A} x_{i j k} * M \quad \forall i \in D, k \in K, \\
& s_{i}-\sum_{k \in K} \sum_{j \in D} x_{i j k} y_{j k}>0 \quad \forall i \in C, \\
& \sum_{i \in D} y_{i k}<c_{k} \quad \forall k \in K, \\
& y_{i k}<c_{k} * \sum_{j \in A} x_{i j k} \quad \forall i \in D, k \in K, \\
& \sum_{k \in K} y_{i k}+U_{i}-d_{i} \geq 0 \quad \forall i \in D, \\
& x_{i j k}=\{0,1\}
\end{aligned}
$$$$
\text { ( } \forall i \in A, j \in A \text {, but } i, j \text { do not belong to } C
$$

at the same time),

$$
y_{i k}, \delta_{i k}, T_{i k}>0 \quad \forall i \in D, k \in K .
$$

Notice that $M$ is a positive number, arbitrarily large. In this model, the objective function (2) seeks to maximize the utilization of vehicles and minimize the delay time. Constraint (3) ensures that the number of vehicles which started from warehouse is less than the number of vehicles existing in warehouses. Constraint (4) ensures that there is at least one vehicle serving each demand node. Constraint (5) represents that a vehicle from the warehouse should finally return to 
the warehouse. Constraint (6) requests that the vehicles must leave as soon as possible after arriving, and each vehicle arrives at a demand node no more than once. Constraint (7) ensures that a vehicle leaving from one demand node could not return to the same node again. Constraint (8) limits the arrival time and delay time at warehouses to 0 . Constraints (9) and (10) impose the restriction on the arrival time and travel time between two demand nodes. Constraint (11) represents the relationship between the arrival time, delay time, and the latest arrival time of a demand node. Constraint (12) limits the delay time to 0 , when the vehicle does not go through the demand node. Constraint (13) ensures that the total amount of relief supplies provided by warehouse $i$ is less than the capacity of warehouse $i$. Constraint (14) ensures that the loading amount of each vehicle is less than the capacity of vehicle $k$. Constraint (15) limits the amount of relief supplies to 0 , if the vehicle does not go through the demand node. Constraint (16) limits the constraints among the demand of grain, the grain delivered, and the unmet demand. Constraints (17) and (18) impose the restriction on the decision variables.

\subsection{Robust Grain Emergency Vehicle Scheduling Problem.} Due to the highly unpredictable reasons, emergencies may cause high uncertainty on traffic. This will seriously affect the vehicle travel time, and it is very different with the general predictive value. For example, earthquake or other natural disasters may cause damage to the road; people's panic may cause excessive traffic congestion, resulting in traffic time being prolonged. On the other hand, due to effective emergency measures, the transportation time will be shortened if the relief supplies are given priority to. Besides, the demand of grain at each point, usually related to the number of the wounded, is difficult to estimate accurately. We can only make a rough estimate according to the historical information. Therefore, for grain emergency vehicle scheduling problem we now consider the transport time and demand uncertainty.

After the occurrence of unexpected events, the travel time from the warehouse to the demand node and the demand of each point cannot be determined accurately, neither can its probability distribution. So we use a robust method, making the travel time and demand belong to a bounded set $U$. As a result, this model is more suitable for realistic situation.

In consideration of the computability of the robust counterpart problem, which is transformed from the deterministic model, we choose a closed bounded convex set as the set of uncertainty. For example, $U_{D}=\left\{\mathbf{d} \mid \mathbf{d}^{0}+\rho \sum_{s=1}^{m} y_{s} \widehat{\mathbf{d}^{s}}, y \in Y\right\}$, where $\rho(\rho>0)$ is the level of uncertainty, $\mathbf{d}^{0}$ is the nominal value of demand, $\widehat{\mathbf{d}^{s}}$ is the scenario vectors, and $\mathbf{y}_{s}$ is the weight of scenario vectors.

Specifically, $U_{D}$ can be described as the following three sets:

$$
\begin{array}{r}
\text { convex hull: } U_{D_{1}}=\left\{\mathbf{d} \mid \mathbf{d}^{0}+\rho \sum_{s=1}^{m} y_{s} \widehat{\mathbf{d}}^{s}, \mathbf{y} \in R^{m},\right. \\
\left.\mathbf{y} \geq 0, \sum_{s=1}^{m} y_{s} \leq 1\right\},
\end{array}
$$

$$
\begin{gathered}
\text { box: } U_{D_{2}}=\left\{\mathbf{d} \mid \mathbf{d}^{0}+\rho \sum_{s=1}^{m} y_{s} \widehat{\mathbf{d}}^{s}, \mathbf{y} \in R^{m},\|\mathbf{y}\|_{\infty} \leq 1\right\}, \\
\text { ellipsoidal: } U_{D_{3}}=\left\{\mathbf{d} \mid \mathbf{d}^{0}+\rho \sum_{s=1}^{m} y_{s} \widehat{\mathbf{d}^{s}},\right. \\
\left.\mathbf{y} \in R^{m}, \mathbf{y}^{T} \mathbf{Q y} \leq \Omega^{2}\right\},
\end{gathered}
$$

where $\mathbf{Q}$ is a positive definite matrix. The weight of scenario vectors $\mathbf{y}_{s}$ belongs to a bounded set $\mathbf{Y}_{i}$ for $i=1,2,3$.

We now propose the robust counterpart problem for grain emergency vehicle scheduling problem with both time and demand uncertainty belonging to a box uncertainty set $U$. Recall that we consider the problem only with uncertainty in constraints (9), (10), and (16).

Proposition 1. If the uncertainty set is $U_{D_{2}}$, the robust corresponding model to the model above can be obtained through the use of constraint (20) instead of constraints (9) and (10), where $t_{i j k}^{0}$ is the nominal value of travel time:

$$
\begin{aligned}
& {\left[M *\left(x_{i j k}-1\right)+\rho \sum_{s=1}^{m}|\widehat{t} \hat{s i j k}|\right]} \\
& <T_{i k}+t_{i j k}{ }^{0}-T_{j k} \\
& <\left[M *\left(1-x_{i j k}\right)-\rho \sum_{s=1}^{m}\left|\widehat{t_{i j k}^{s}}\right|\right] \\
& \forall i \in C, \quad j \in D, \quad k \in K, \\
& {\left[M *\left(x_{i j k}-1\right)+\rho \sum_{s=1}^{m}|\widehat{t} \hat{s i k k}|\right]} \\
& <T_{i k}+t_{i j k}{ }^{0}-T_{j k} \\
& <\left[M *\left(1-x_{i j k}\right)-\rho \sum_{s=1}^{m}\left|\widehat{t_{i j k}^{s}}\right|\right] \\
& \forall i \in D, \quad j \in D, \quad k \in K \text {. }
\end{aligned}
$$

Proof. Using the definition of $Y_{2}$ we can write $\sup _{y \in \mathbf{Y}_{2}} \mathbf{y}^{T} \mathbf{D}_{j}$ and its dual as the following pair of LPs:

$$
\begin{aligned}
& \text { (Primal) } \max \mathbf{y}^{T} \mathbf{D}_{j} . \quad \text { s.t. } \mathbf{y} \leq \mathbf{e}, \mathbf{y} \geq-\mathbf{e}, \\
& \text { (Dual) } \min \mathbf{e}^{T}(\boldsymbol{\alpha}+\boldsymbol{\beta}), \quad \text { s.t. } \boldsymbol{\alpha}-\boldsymbol{\beta}=\mathbf{D}_{j}, \boldsymbol{\alpha}, \boldsymbol{\beta} \geq 0 \text {. }
\end{aligned}
$$

It is simple to verify that the optimal solution to the dual problem will satisfy $\alpha_{k}^{*}+\beta_{k}^{*}=\rho \sum_{s=1}^{m}\left|\widehat{t_{i j k}^{s}}\right|$ for every $k=1, \ldots, q$. Therefore, the dual optimal objective value is $\rho \sum_{k=1}^{q} \sum_{s=1}^{m}\left|\widehat{t_{i j k}^{s}}\right|$. Enforcing the robust feasibility condition 
on (9) and (10) with the above optimal dual objective value we obtain constraints (20). (22):

Similarly, we can replace constraint (16) by constraint

$$
\sum_{k \in K} y_{i k}+U_{i}-d_{i}^{0} \geq \rho \sum_{s=1}^{m} \widehat{d_{i}^{s}} \quad \forall i \in D .
$$

\section{Experimental Analysis}

From the part above, we can see that the transformed robust model is still 0-1 mixed integer programming. Although the $0-1$ mixed integer programming could not be solved by polynomial time algorithm, small scale problems can still use the exact algorithm such as the branch and bound method. We first use the branch and bound algorithm to solve the deterministic model with many warehouses.

Assume that there are three warehouses and eight demand nodes. The total amount of relief grain provided by each warehouse is 1100,1800 , and 2700 , and the number of vehicles available in each warehouse is 1,2 , and 3 . The capacity of vehicle is 1000 . The transportation time between two demand nodes is assumed to be symmetric. Then we generate 8 integers randomly between $[100,1000]$ as the demand at each node, and another 8 integers between $[1,10]$ as the latest time relief supplies arrived at demand node. Last, generate integers between $[1,10]$ as the travel time between every two nodes (see Table 1, unit: hours).

To solve this deterministic problem, we use the linear interactive and general optimizer (LINGO). The results are as follows (see Table 2).

From Table 1, we can see that, with the weight becoming larger, both the total amount of utilization rate of vehicle and the total delay time will be increased. Therefore, the size of the weight depends on which factor the decision maker pays more attention to.

Next, we will analyze the influence of travel time and demand on the optimal vehicle route. Here we assume that the weight of the utilization of vehicles is 1 .

When the travel time changes slightly, such as the travel time between warehouse 2 , and the demand node 1 changes to 5 hours, the optimal route becomes 3-6-7-4-11-1 and 3-59-10-3-8-2. The route has changed greatly. Therefore, we can conclude that the optimal solution is very sensitive to changes in travel time.

When we change the demand of the demand node 2 from 450 to 500 , the optimal route becomes 3-6-5-8-2, 3-6-7-411-1, and 3-5-9-10-2. The result is different with the former one. Thus, the optimal solution is sensitive to changes in both travel time and demand.

Now we have a feasibility test on the original result. Then we find that when the demand or travel time changes, the original optimal route becomes infeasible.

Through the experiment above, we can see that when the travel time between demand nodes or the demand at each node is slightly changed, the final optimal route will also be changed (see Table 3 ). This indicates that the optimal solution is very sensitive to the travel time and demand. Due to various factors, when the incident occurs, we cannot know the exact and accurate demand and travel time between demand nodes. Thus, there is probably some deviation from the original estimates. If we still calculate the route according to the original estimate, this may cause larger utilization rate of vehicle or increased delay time. Besides, even the requirements of feasibility cannot be met, resulting in more serious consequences.

Then we first have a test on the robust counterpart of grain emergency vehicle scheduling problem. Here we choose the box constraints for testing. We consider a box uncertainty set for the travel time. It is given by $t_{i j k} \in\left[t_{i j k}^{0}-\rho \widehat{t_{i j k}}, t_{i j k}^{0}+\rho \widehat{t_{i j k}}\right]$, where $t_{i j k}^{0}$ are the nominal values and $\widehat{t_{i j k}}$ are the deviation values. We use the data from Table 1 as the nominal values of travel time (when $\gamma=10$ ).

Then we generate integers randomly between $[0,5]$ as the deviation values of travel time between nodes.

Next, let us consider the changes of objective function when the travel time is uncertain. To solve this model with time uncertainty, we use the linear interactive and general optimizer (LINGO). The optimal solutions under different values of $\rho$ are as follows (see Table 4).

When the level of uncertainty $\rho$ changes, the optimal routes change as well. Although the delay time changes, the utilization rate of vehicle does not change. With a box uncertainty set for the travel time, we use the optimal solution for the nominal value to have the feasibility test. We conclude that the optimal routes seeking out from the nominal value are sometimes infeasible. Through the experiment, it can be obtained that the optimal solution of deterministic model is sensitive to the travel time. When the travel time deviates from the nominal value, the optimal solution for the nominal value is not feasible sometimes, but the optimal solution obtained by the robust model is not sensitive to the travel time so as to maintain the robust solutions.

Secondly, we use LINGO to calculate the optimal solution with uncertain demand under different values of $\rho$. The results are as follows (see Table 5).

When the level of uncertainty $\rho$ changes, the optimal routes change as well. With the change of demand, the utilization rate of vehicles and the delay time change as well, which eventually leads to the inconsistency of objective function. From the results we can see that, with the level of uncertainty increasing, the utilization rate of vehicles reduced and delay time increased, which also result in the objective function being reduced. So we can conclude that we should try to improve the accuracy of demand forecasting and reduce the fluctuation of demand.

With a box uncertainty set for the demand, we use the optimal solution for the nominal value to have the feasibility test. We conclude that the optimal routes seeking out from the nominal value are sometimes infeasible. Through the experiment, it can be obtained that the optimal solution of deterministic model is sensitive to the travel time. When the travel time deviates from the nominal value, the optimal solution for the nominal value is not feasible sometimes, but the optimal solution obtained by the robust model is not sensitive to the travel time so as to maintain the robust solutions. 
TABLE 1: The travel time between every two nodes.

\begin{tabular}{|c|c|c|c|c|c|c|c|c|c|c|c|}
\hline & W1 & W2 & W3 & DN1 & DN2 & DN3 & DN4 & DN5 & DN6 & DN7 & DN8 \\
\hline $\mathrm{W} 1$ & - & 1 & 2 & 4 & 3 & 6 & 3 & 5 & 9 & 7 & 6 \\
\hline W2 & 1 & - & 3 & 2 & 5 & 7 & 6 & 3 & 4 & 8 & 1 \\
\hline W3 & 2 & 3 & - & 4 & 2 & 1 & 2 & 7 & 5 & 6 & 8 \\
\hline DN1 & 4 & 2 & 4 & - & 5 & 8 & 1 & 6 & 3 & 7 & 1 \\
\hline DN2 & 3 & 5 & 2 & 5 & - & 2 & 4 & 2 & 1 & 5 & 3 \\
\hline DN3 & 6 & 7 & 1 & 8 & 2 & - & 1 & 5 & 4 & 8 & 1 \\
\hline DN4 & 3 & 6 & 2 & 1 & 4 & 1 & - & 3 & 7 & 4 & 9 \\
\hline DN5 & 5 & 3 & 7 & 6 & 2 & 5 & 3 & - & 9 & 4 & 3 \\
\hline DN6 & 9 & 4 & 5 & 3 & 1 & 4 & 7 & 9 & - & 2 & 8 \\
\hline DN7 & 7 & 8 & 6 & 7 & 5 & 8 & 4 & 4 & 2 & - & 7 \\
\hline DN8 & 6 & 1 & 8 & 1 & 3 & 1 & 9 & 3 & 8 & 7 & - \\
\hline
\end{tabular}

W: warehouse; DN: demand node.

The demand generated randomly: 700, 450, 900, 300, 700, 450, 750, and 600

The latest arrival time generated randomly: 0, 0, 0, 5, 4, 7, 6, and 9 .

TABLE 2: Vehicle scheduling under different weight.

\begin{tabular}{|c|c|c|c|}
\hline Weight & $\gamma=1$ & $\gamma=10$ & $\gamma=100$ \\
\hline Vehicle 1 & - & $1-5-3$ & $1-5-9-1$ \\
\hline Vehicle 2 & - & $2-11-4-1$ & $2-4-1$ \\
\hline Vehicle 3 & - & $2-11-6-1$ & $2-11-1$ \\
\hline Vehicle 4 & $3-6-11-4-7-1$ & $3-7-8-3$ & $3-6-8-1$ \\
\hline Vehicle 5 & $3-5-8-1$ & $3-5-9-10-1$ & $3-6-7-3$ \\
\hline Vehicle 6 & $3-6-5-9-10-3$ & $3-6-9-10-2$ & $3-10-1$ \\
\hline \multicolumn{4}{|c|}{ Utilization rate of vehicle } \\
\hline Detail & $\begin{array}{c}r_{1}=0 \%, r_{2}=0 \% \\
r_{3}=0 \%, r_{4}=70 \%, \\
r_{5}=100 \%, r_{6}=100 \%\end{array}$ & $\begin{array}{l}r_{1}=35 \%, r_{2}=100 \%, \\
r_{3}=80 \%, r_{4}=100 \%, \\
r_{5}=100 \%, r_{6}=70 \%\end{array}$ & $\begin{array}{c}r_{1}=90 \%, r_{2}=70 \%, \\
r_{3}=60 \%, r_{4}=100 \%, \\
r_{5}=90 \%, r_{6}=75 \%\end{array}$ \\
\hline Total & 2.70 & 4.85 & 4.85 \\
\hline \multicolumn{4}{|l|}{ Delay time } \\
\hline Detail & 0 & $\begin{array}{l}\delta_{4,2}=2, \delta_{5,1}=3, \\
\delta_{6,3}=2, \delta_{10,5}=1, \\
\delta_{11,2}=1, \delta_{11,3}=1\end{array}$ & $\begin{array}{c}\delta_{4,2}=2, \delta_{5,1}=3, \\
\delta_{8,4}=1, \delta_{9,1}=4, \\
\delta_{11,3}=1\end{array}$ \\
\hline Total & $\mathbf{0}$ & 10 & 11 \\
\hline Objective function & 2.70 & 47.5 & 474 \\
\hline
\end{tabular}

TABLE 3: Random deviation of travel time.

\begin{tabular}{|c|c|c|c|c|c|c|c|c|c|c|c|}
\hline & W1 & W2 & W3 & DN1 & DN2 & DN3 & DN4 & DN5 & DN6 & DN7 & DN8 \\
\hline W1 & 0 & 1 & 2 & 2 & 0 & 3 & 1 & 0 & 4 & 3 & 1 \\
\hline W2 & 1 & 0 & 3 & 1 & 3 & 2 & 1 & 2 & 1 & 5 & 0 \\
\hline W3 & 2 & 3 & 0 & 1 & 2 & 1 & 2 & 4 & 2 & 1 & 2 \\
\hline DN1 & 2 & 1 & 1 & 0 & 2 & 4 & 1 & 1 & 2 & 3 & 1 \\
\hline DN2 & 0 & 3 & 2 & 2 & 0 & 2 & 0 & 2 & 1 & 0 & 3 \\
\hline DN3 & 3 & 2 & 1 & 4 & 2 & 0 & 1 & 2 & 1 & 4 & 1 \\
\hline DN4 & 1 & 1 & 2 & 1 & 0 & 1 & 0 & 0 & 2 & 1 & 3 \\
\hline DN5 & 0 & 2 & 4 & 1 & 2 & 2 & 0 & 0 & 5 & 1 & 2 \\
\hline DN6 & 4 & 1 & 2 & 2 & 1 & 1 & 2 & 5 & 0 & 0 & 5 \\
\hline DN7 & 3 & 5 & 1 & 3 & 0 & 4 & 1 & 1 & 0 & 0 & 1 \\
\hline DN8 & 1 & 0 & 2 & 1 & 3 & 1 & 3 & 2 & 5 & 1 & 0 \\
\hline
\end{tabular}


TABLE 4: Vehicle routes under different $\rho$ with travel time uncertainty.

\begin{tabular}{|c|c|c|c|}
\hline Uncertainty & $\rho=1.5$ & $\rho=2.0$ & $\rho=3.0$ \\
\hline Vehicle 1 & $1-4-1$ & $1-6-1$ & $1-6-1$ \\
\hline Vehicle 2 & $2-8-1$ & $2-4-2$ & $2-10-2$ \\
\hline Vehicle 3 & $2-10-1$ & $2-10-1$ & $2-4-2$ \\
\hline Vehicle 4 & $3-4-11-6-7-1$ & $3-9-11-7-1$ & $3-5-9-1$ \\
\hline Vehicle 5 & $3-7-8-5-11-1$ & $3-5-8-10-1$ & $3-8-10-4-5-9-2$ \\
\hline Vehicle 6 & $3-6-9-5-3$ & $3-8-11-10-1$ & $3-6-11-4-8-7-1$ \\
\hline \multicolumn{4}{|c|}{ Utilization rate of vehicle } \\
\hline Detail & $\begin{array}{c}r_{1}=70 \%, r_{2}=70 \% \\
r_{3}=75 \%, r_{4}=70 \% \\
r_{5}=100 \%, r_{6}=100 \%\end{array}$ & $\begin{array}{l}r_{1}=90 \%, r_{2}=70 \% \\
r_{3}=55 \%, r_{4}=100 \% \\
r_{5}=100 \%, r_{6}=70 \%\end{array}$ & $\begin{array}{c}r_{1}=90 \%, r_{2}=75 \%, \\
r_{3}=50 \%, r_{4}=70 \%, \\
r_{5}=100 \%, r_{6}=100 \%\end{array}$ \\
\hline Total & 4.85 & 4.85 & 4.85 \\
\hline \multicolumn{4}{|l|}{ Delay time } \\
\hline Detail & $\begin{array}{c}\delta_{4,1}=1, \delta_{8,2}=0.1 \\
\delta_{10,3}=0.5\end{array}$ & $\begin{array}{c}\delta_{4,2}=0.1, \delta_{6,1}=0.1 \\
\delta_{10,3}=0.1\end{array}$ & $\delta_{4,3}=0.1, \delta_{6,1}=0.1$ \\
\hline Total & 1.6 & 0.3 & 0.2 \\
\hline Objective function & 46.9 & 48.3 & 48.2 \\
\hline
\end{tabular}

TABLE 5: Vehicle routes under different $\rho$ with travel demand uncertainty.

\begin{tabular}{|c|c|c|c|}
\hline Uncertainty & $\rho=1.5$ & $\rho=2.0$ & $\rho=3.0$ \\
\hline Vehicle 1 & - & - & - \\
\hline Vehicle 2 & $2-4-2$ & $2-11-2$ & $2-11-1$ \\
\hline Vehicle 3 & $2-11-6-1$ & $2-11-6-1$ & $2-4-1$ \\
\hline Vehicle 4 & $3-6-9-1$ & $3-5-9-10-1$ & $3-5-8-1$ \\
\hline Vehicle 5 & $3-5-8-1$ & $3-5-8-3$ & $3-6-7-4-11-2$ \\
\hline Vehicle 6 & $3-7-10-1$ & $3-6-7-4-11-8-3$ & $3-9-10-3$ \\
\hline \multicolumn{4}{|c|}{ Utilization rate of vehicle } \\
\hline Detail & $\begin{array}{c}r_{1}=0 \%, r_{2}=62.5 \% \\
r_{3}=100 \%, r_{4}=72.5 \% \\
r_{5}=100 \%, r_{6}=90 \%\end{array}$ & $\begin{array}{c}r_{1}=0 \%, r_{2}=35 \% \\
r_{3}=100 \%, r_{4}=100 \% \\
r_{5}=70 \%, r_{6}=100 \%\end{array}$ & $\begin{array}{c}r_{1}=0 \%, r_{2}=35 \%, \\
r_{3}=100 \%, r_{4}=100 \%, \\
r_{5}=65 \%, r_{6}=100 \%\end{array}$ \\
\hline Total & 4.25 & 4.05 & 4.00 \\
\hline \multicolumn{4}{|l|}{ Delay time } \\
\hline Detail & $\delta_{4,3}=2, \delta_{11,2}=1$ & $\begin{array}{c}\delta_{6,3}=2, \delta_{11,2}=2 \\
\delta_{11,3}=1\end{array}$ & $\begin{array}{c}\delta_{4,2}=2, \delta_{6,3}=2 \\
\delta_{11,3}=1\end{array}$ \\
\hline Total & 3 & 5 & 5 \\
\hline Objective function & 46.9 & 39.5 & 35.5 \\
\hline
\end{tabular}

The examples above provide some management measures for the decision makers. Firstly, it is very important for them to make a trade-off between the utilization rate of vehicles and the delay time. As shown in Table 2, the weight has great influence on the objective function. The managers should determine the weight according to different situations and the importance of the two factors. Secondly, they should locate the warehouses properly to make the distance shorter. It is a good way to reduce the delay time. In addition, they should also use some technology to make a better prediction of travel time and demand. This will also reduce the complexity of the model.

\section{Conclusions}

Emergency logistics is a new research field, which has good application background and broad perspective. This paper mainly studies the grain emergency vehicle scheduling optimization problems. The research is summarized as follows.

(1) We analyze the characteristics of grain emergency logistics and the significance of grain emergency vehicle scheduling problem based on the analysis and reference of the relevant theory.

(2) According to the specific characteristics of emergency logistics, we established the mathematical model of 
grain emergency vehicle scheduling model. On the basis of research on grain emergency logistics vehicle scheduling problem, the question is discussed, and corresponding mathematical models are established.

(3) Then we consider the uncertainty in travel time and demand and present the robust counterpart model. We propose the uncertainty belonging to a box uncertainty set. Our study shows that the robust optimization is an effective method to solve the vehicle scheduling problem with uncertainty, because it is not necessary to know the amount of uncertainty distribution function in advance.

(4) We use LINGO to solve the two models. Besides, we use an example to analyze the model. Example shows that the robust method is feasible and effective in solving grain emergency vehicle scheduling problem and optimization. Our computational results show that the chance constrained model can be more or less efficient than the robust model depending on the problem parameters and uncertainty assumptions. Lastly, we put forward some management measures to improve the logistics system when uncertainties occur.

However, future work is still needed to solve the grain emergency vehicle scheduling model. Further work may consider additional uncertainty sets such as convex hull and ellipsoidal uncertainty. These sets has been proved to solve to larger problems while restricting the uncertainty away from worst-case scenarios.

\section{Conflict of Interests}

The authors declare that there is no conflict of interests regarding the publication of this paper.

\section{References}

[1] G. B. Dantzig and J. H. Ramser, "The truck dispatching problem," Management Science, vol. 6, no. 1, pp. 80-91, 1959.

[2] A. K. Rathi, R. S. Solanki, and R. L. Church, "Allocating resources to support a multicommodity flow with time windows,” Oak Ridge National Lab, Oak Ridge, Tenn, USA, 1990.

[3] F. Fiedrich, F. Gehbauer, and U. Rickers, "Optimized resource allocation for emergency response after earthquake disasters," Safety Science, vol. 35, no. 1-3, pp. 41-57, 2000.

[4] J. C. Morales, Planning robust freight transportation operations [Dissertation], 2006.

[5] A. L. Soyster, "Convex programming with set-inclusive constraints and applications to inexact linear programming," Operations Research, vol. 21, pp. 1154-1157, 1973.

[6] J. M. Mulvey, R. J. Vanderbei, and S. A. Zenios, "Robust optimization of large-scale systems," Operations Research, vol. 43, no. 2, pp. 264-281, 1995.

[7] A. Ben-Tal and A. Nemirovski, "Robust solutions of uncertain linear programs," Operations Research Letters, vol. 25, no. 1, pp. $1-13,1999$.

[8] A. Ben-Tal and A. Nemirovski, "Robust solutions of linear programming problems contaminated with uncertain data," Mathematical Programming B, vol. 88, no. 3, pp. 411-424, 2000.
[9] A. Ben-Tal and A. Nemirovski, "Robust optimizationmethodology and applications," Mathematical Programming B, vol. 92, no. 3, pp. 453-480, 2002.

[10] L. El Ghaoui and H. Lebret, "Robust solutions to least-squares problems with uncertain data," SIAM Journal on Matrix Analysis and Applications, vol. 18, no. 4, pp. 1035-1064, 1997.

[11] L. El Ghaoui, F. Oustry, and H. Lebret, "Robust solutions to uncertain semidefinite programs," SIAM Journal on Optimization, vol. 9, no. 1, pp. 33-52, 1999.

[12] D. Bertsimas and M. Sim, "Robust discrete optimization and network flows," Mathematical Programming, vol. 98, no. 1-3, pp. 49-71, 2003.

[13] D. Bertsimas, D. Pachamanova, and M. Sim, "Robust linear optimization under general norms," Operations Research Letters, vol. 32, no. 6, pp. 510-516, 2003.

[14] D. Bertsimas and M. Sim, “The price of robustness," Operations Research, vol. 52, no. 1, pp. 35-53, 2004. 


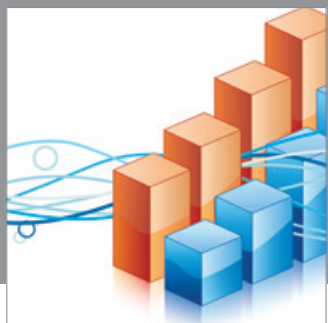

Advances in

Operations Research

mansans

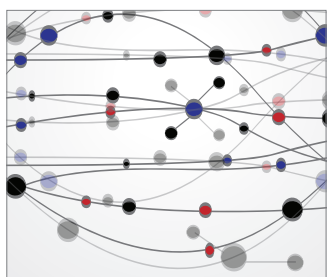

The Scientific World Journal
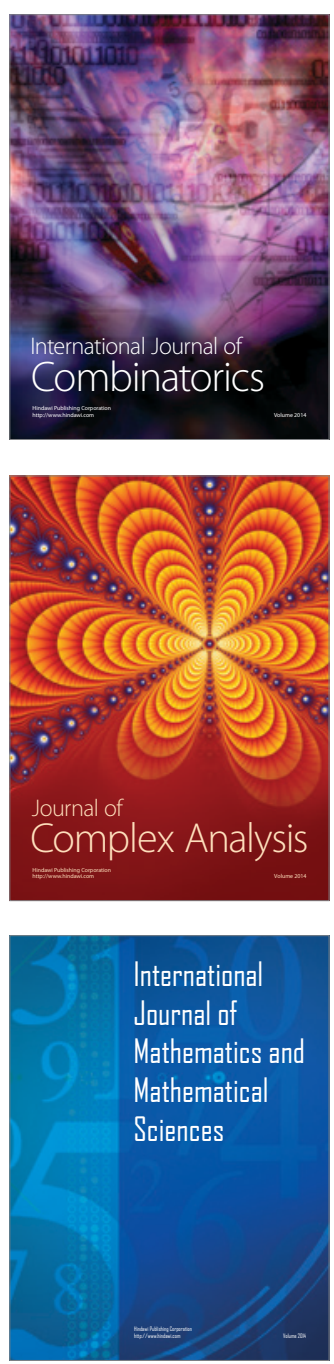
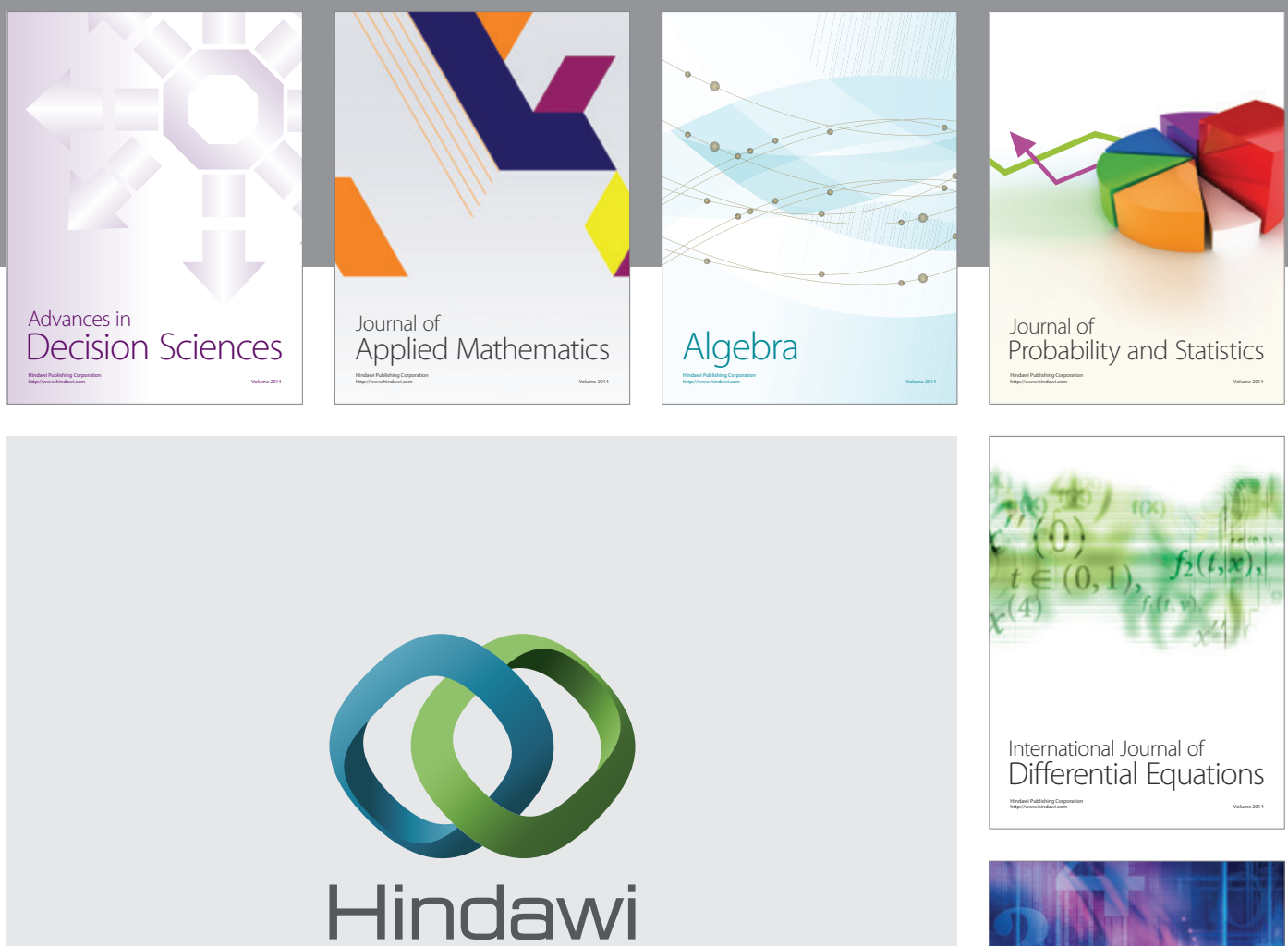

Submit your manuscripts at http://www.hindawi.com
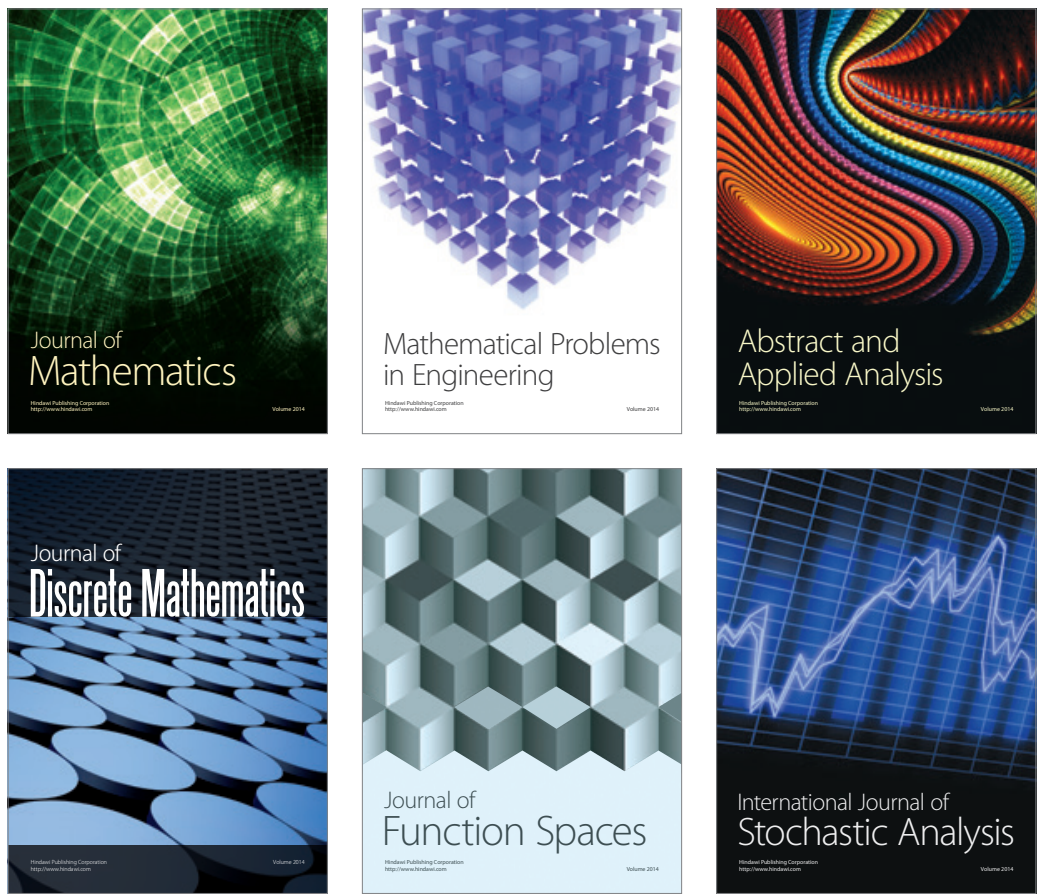

Journal of

Function Spaces

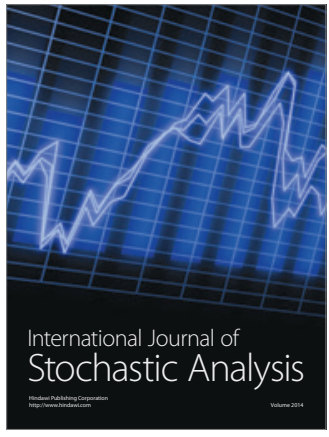

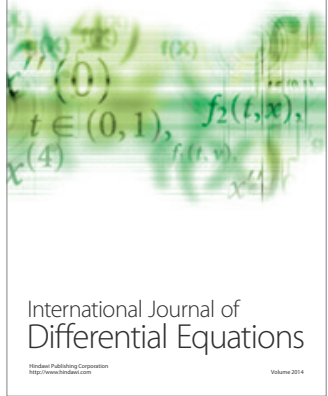
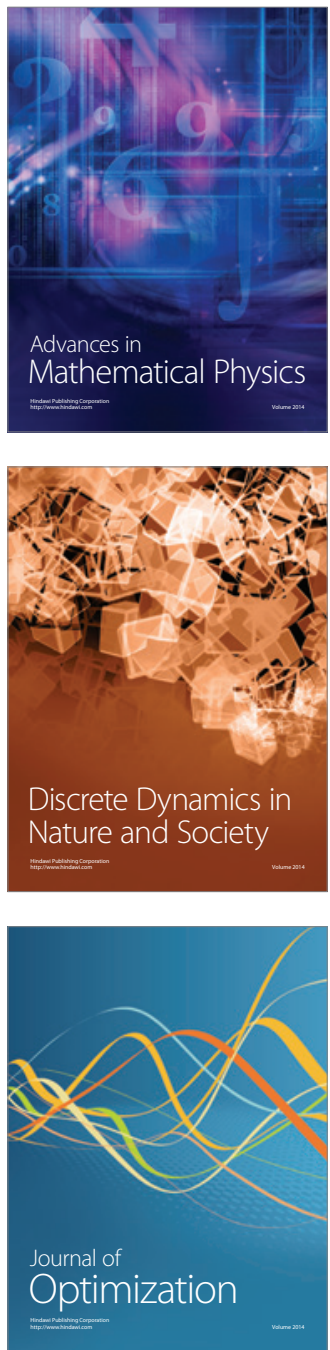